\title{
Combined BRAF and MEK Inhibition with Vemurafenib and Cobimetinib for Patients with Advanced Melanoma
}

\author{
Antonio M Grimaldi, Ester Simeone, Lucia Festino, Vito Vanella and Paolo A Ascierto \\ Melanoma, Cancer Immunotherapy and Innovative Therapy Unit, Istituto Nazionale Tumori Fondazione "G. Pascale", Napoli, Italy
}

\section{A} cquired resistance is the most common cause of BRAF inhibitor monotherapy treatment failure, with the majority of patients experiencing disease progression with a median progression-free survival of 6-8 months. As such, there has been considerable focus on combined therapy with dual BRAF and MEK inhibition as a means to improve outcomes compared with monotherapy. In the COMBI-d and COMBI-v trials, combined dabrafenib and trametinib was associated with significant improvements in outcomes compared with dabrafenib or vemurafenib monotherapy, in patients with BRAF-mutant metastatic melanoma. The combination of vemurafenib and cobimetinib has also been investigated. In the phase III CoBRIM study in patients with unresectable stage III-IV BRAF-mutant melanoma, treatment with vemurafenib and cobimetinib resulted in significantly longer progression-free survival and overall survival (OS) compared with vemurafenib alone. One-year OS was $74.5 \%$ in the vemurafenib and cobimetinib group and $63.8 \%$ in the vemurafenib group, while 2 -year OS rates were $48.3 \%$ and $38.0 \%$, respectively. The combination was also well tolerated, with a lower incidence of cutaneous squamous-cell carcinoma and keratoacanthoma compared with monotherapy. Dual inhibition of both MEK and BRAF appears to provide a more potent and durable anti-tumour effect than BRAF monotherapy, helping to prevent acquired resistance as well as decreasing adverse events related to BRAF inhibitor-induced activation of the MAPK-pathway. Combined BRAF and MEK inhibition is the standard of care in patients with advanced BRAF-mutant melanoma.

\section{Keywords}

Metastatic melanoma, BRAF inhibitors, MEK inhibitors, vemurafenib, cobimetinib, combination therapy

Disclosure: Antonio M Grimaldi received honoraria from BMS, MSD, Novartis and Roche Genentech, and had consultant/advisory role for MSD and Novartis. Ester Simeone recevied honoraria from BMS, Novartis and Roche Genentech. Ester Simeone had consultant/ advisory role for BMS. Lucia Festino and Vito Vanella have nothing to disclose in relation to this article. Paolo $\mathrm{A}$ Ascierto received research founding form BMS, Roche Genentech, Array Biopharma, and had consulting/ advisory role for BMS, Roche Genentech, MSD, Novartis, Array Biopharma, Merck Serono and Pierre Fabre. This study involves a review of the literature and did not involve any studies with human or animal subjects performed by any of the authors. No funding was received for the publication of this article.

Authorship: All named authors meet the International Committee of Medical Journal Editors (ICMJE) criteria for authorship of this manuscript, take responsibility for the integrity of the work as a whole, and have given final approval to the version to be published.

open Access: This article is published under the Creative Commons Attribution Noncommercial License which permits any non-commercial use, distribution, adaptation and reproduction provided the original author(s) and source are given appropriate credit.

Received: 17 October 2016

Accepted: 18 January 2017

Citation: European Oncology \& Haematology, 2017;13(1):61-5

Corresponding Author: Paolo A Ascierto, Melanoma, Cancer Immunotherapy and Innovative Therapy Unit, Istituto Nazionale Tumori Fondazione "G. Pascale",

Via Mariano Semmola, 80131 Napoli, Italy.

E: paolo.ascierto@gmail.com
Metastatic melanoma management has been transformed in the past few years, with the development of new therapeutic options that provide significant survival benefits replacing palliative chemotherapy. One aspect of this advance has been an increased understanding of molecular aberrations in melanoma, in particular, those associated with the mitogen-activated protein kinase (MAPK) pathway. This has resulted in the identification of new oncogenic targets and the development of targeted therapies, including the BRAF inhibitors, vemurafenib and dabrafenib, and the MEK inhibitors, trametinib and cobimetinib.

Around $40-60 \%$ of cutaneous melanomas have mutations in BRAF that lead to constitutive activation of downstream signalling through the MAPK pathway. ${ }^{1}$ The majority of BRAF mutations are V600E (glutamic acid substituted for valine), while 10\% are V600K mutations with the remainder involving rare mutations in V600D, V600R or V600M. BRAF V600 mutations significantly increase the catalytic activity of the BRAF protein, leading to constitutive activation and phosphorylation of MEK and ERK in the RAS-RAF-MAPK signalling cascade. ${ }^{2}$ BRAF inhibitors and MEK inhibitors specifically inhibit signal transduction by respectively targeting mutated BRAF or MEK.

\section{BRAF inhibitors}

The first BRAF inhibitor to be approved for unresectable or metastatic BRAF V600-mutant melanoma was vemurafenib in 2011. In the phase III BRIM-3 trial of 675 patients with previously untreated metastatic BRAF V600E-mutant melanoma, vemurafenib $960 \mathrm{mg}$ twice daily resulted in longer progression-free survival (PFS) (5.3 versus 1.6 months) and overall survival (OS) (13.6 versus 9.7 months) compared to chemotherapy with dacarbazine. ${ }^{3}$ At a median follow up of 7 months, the hazard ratio (HR) for death in the vemurafenib group was 0.37 ( $95 \%$ confidence interval $[\mathrm{Cl}$, $0.2-0.55 ; p<0.001$ ) while the HR for tumour progression was 0.26 ( $95 \% \mathrm{Cl}, 0.20-0.33 ; p<0.001$ ). In a later analysis with a median follow-up of 12.5 months on vemurafenib and 9.5 months on dacarbazine, vemurafenib maintained significantly longer median PFS (6.9 versus 1.6 months; HR 0.38 [95\% Cl 0.32-0.46]; $\mathrm{p}<0.0001$ ) and median OS (13.6 versus 9.7 months; HR 0.70 [95\% $\mathrm{Cl}$ 0.57-0.87]; $\mathrm{p}=0.0008$ ). ${ }^{4} \mathrm{~A}$ second BRAF inhibitor, dabrafenib, was approved in 2013 having achieved a similar improvement in PFS when compared with dacarbazine (5.1 versus 2.7 months; HR 0.30 [95\% Cl, 0.18-0.51; p<0.0001) in the phase III BREAK-3 trial. ${ }^{5} \mathrm{~A}$ third BRAF inhibitor, encorafenib (LGX 818) is also in development. 
Table 1: Combined BRAF and MEK inhibition studies in melanoma

\begin{tabular}{|c|c|c|c|c|}
\hline & Overall response rate & $\begin{array}{l}\text { Median } \\
\text { duration of } \\
\text { response }\end{array}$ & $\begin{array}{l}\text { Median progression-free } \\
\text { survival }\end{array}$ & Overall survival \\
\hline \multicolumn{5}{|l|}{ Dabrafenib + trametinib } \\
\hline $\begin{array}{l}\text { Dabrafenib + trametinib } \\
\text { versus dabrafenib (Flaherty } \\
2012^{11} \text { ) }\end{array}$ & $76 \%$ versus $54 \% ; p=0.03$ & 10.5 versus 5.6 & $\begin{array}{l}9.4 \text { versus } 5.8 \text { (HR 0.39; } 95 \% \mathrm{Cl} \text { : } \\
0.25-0.62 ; \mathrm{p}<0.001)\end{array}$ & $\begin{array}{l}\text { Median not reached at the time of this analysis. } \\
\text { 1-year os: } 79 \% \text { versus } 70 \% \text { ( } 80 \% \text { of patients in } \\
\text { the monotherapy group crossed over to the } \\
\text { combination group at disease progression) }\end{array}$ \\
\hline $\begin{array}{l}\text { COMBI-d: Dabrafenib + } \\
\text { trametinib versus dabrafenib } \\
\left(\text { Long } 2014^{18} \text {; Long } 2015^{19}\right)\end{array}$ & $67 \%$ versus $51 \% ; p=0.002$ & 9.2 versus 10.2 & $\begin{array}{l}11.0 \text { versus } 8.8(\mathrm{HR} 0.67,95 \% \mathrm{Cl} \\
0.53-0.84 ; p=00004)\end{array}$ & $\begin{array}{l}\text { 25.1 versus } 18.7 \text { (HR } 0.71,95 \% \text { Cl } 0.55-0.92 ; \\
p=0.0107) \\
\text { 1-year OS: } 74 \% \text { versus } 68 \% \\
\text { 2-year OS: } 51 \% \text { versus } 42 \%\end{array}$ \\
\hline $\begin{array}{l}\text { COMBI-v: Dabrafenib } \\
+ \text { trametinib versus } \\
\text { vemurafenib(Robert 2015²1) }\end{array}$ & $64 \%$ versus $51 \% ; p<0.001$ & 13.8 versus 7.5 & $\begin{array}{l}11.4 \text { versus } 7.3 \text { (HR } 0.56 ; 95 \% \\
\mathrm{Cl}: 0.46-0.69 ; \mathrm{p}<0.001)\end{array}$ & $\begin{array}{l}\text { 1-year OS: } 72 \% \text { versus 65\% (HR 0.69; } 95 \% \mathrm{Cl} \\
0.53-0.89 ; p=0.005)\end{array}$ \\
\hline \multicolumn{5}{|l|}{ Vemurafenib + cobimetinib } \\
\hline $\begin{array}{l}\text { BRIM7: Vemurafenib + } \\
\text { cobimetinib (Ribas 2014²3) }\end{array}$ & 87\% (BRAF inhibitor-naïve) & & 13.7 (BRAF inhibitor-naïve) & - \\
\hline $\begin{array}{l}\text { CoBRIM: Vemurafenib } \\
+ \text { cobimetinib versus } \\
\text { vemurafenib (Larkin 20147; } \\
\text { Ascierto } 2016^{26} \text { ) }\end{array}$ & $\begin{array}{l}\text { At } 7.3 \text { months median follow-up: } \\
68 \% \text { versus } 45 \% ; p<0.001 \\
\text { At } 14.2 \text { months median follow-up: } \\
70 \% \text { versus } 50 \% ; p<0.0001\end{array}$ & $\begin{array}{l}\text { At } 14.2 \text { months: } \\
13.0 \text { versus } 9.2 \\
\text { months }\end{array}$ & $\begin{array}{l}\text { At } 7.3 \text { months: } 9.9 \text { versus } \\
6.2 \text { months; } \mathrm{HR} 0.51,95 \% \mathrm{Cl} \\
0.39-0.68 ; \mathrm{p}<0.001 \\
\text { At } 14.2 \text { months: } 12.3 \text { versus } \\
7.2 \text { months (HR } 0.58,95 \% \mathrm{Cl} \\
0.46-0.72, \mathrm{p}<0.0001 \text { ) }\end{array}$ & $\begin{array}{l}\text { At } 14.2 \text { months: } 22.3 \text { versus } 17.4 \text { months (HR } \\
0.70,95 \% \mathrm{Cl} 0.55-0.90 ; \mathrm{p}=0.005) \\
\text { 1-year OS:74.5\% versus } 63.8 \% \\
\text { 2-year OS: } 48.3 \% \text { versus } 38.0 \%\end{array}$ \\
\hline
\end{tabular}

$\mathrm{Cl}=$ confidence interval; $\mathrm{HR}=$ hazard ratio; OS = overall survival.

BRAF inhibitors typically result in a rapid response to treatment, with an early reduction in symptom burden and improved performance status. This appears to be especially marked in patients with the most severe clinical condition (the so-called "Lazarus Effect") and is mediated through a fast metabolic disease shutdown and a slower decrease in the size of metastatic lesions.

BRAF inhibitors are generally well tolerated with the most common toxicities being rash, fatigue and joint pain. Vemurafenib is associated with an increased frequency of photosensitivity than dabrafenib although pyrexia is less frequent. Increased cutaneous squamous-cell carcinoma (SCC) and keratoacanthoma (KA) has been observed with both vemurafenib ( $26 \%$ of patients) and dabrafenib (14\% of patients), usually within the first 2 months of treatment. ${ }^{6,7}$ This toxicity appears to be secondary to the paradoxical activation of the MAPK pathway in keratinocytes together with activation of RAS mutation mediated signaling. ${ }^{8.9}$

\section{MEK inhibitors}

The first MEK inhibitor to be approved for the treatment of advanced BRAF V600 mutant melanoma was trametinib in 2014. In a phase II clinical trial, trametinib showed significant clinical activity in BRAF inhibitor-naïve metastatic melanoma patients previously treated with chemotherapy and/or immunotherapy (median PFS: 4.0 months; $2 \%$ complete response, $23 \%$ partial response). ${ }^{10}$ However, minimal clinical activity was observed when given as sequential therapy in patients previously treated with a BRAF inhibitor (median PFS: 1.8 months; no confirmed responses), suggesting that BRAF inhibitor resistance mechanisms also confer resistance to MEK inhibitors. In the randomised phase III METRIC trial, 322 previously treated (immuno- or chemotherapy) BRAF inhibitor-naïve patients with advanced BRAF-mutant melanoma were randomised to trametinib $2 \mathrm{mg}$ once daily or chemotherapy with dacarbazine or paclitaxel. ${ }^{11}$ Trametinib was associated with improvements in response rate $(22 \%$ versus $8 \%$ ), median PFS ( 4.8 versus 1.5 months; $p<0.001$ ) and 6-month OS (81\% versus 67\%; HR 0.54, p=0.01). Hypertension, rash and fatigue were the most commonly reported grade 3 adverse effects and were manageable by dose interruption and dose reduction. Although approved as monotherapy for the treatment of BRAF V600 mutant metastatic melanoma, trametinib is not often used as such because BRAF inhibitor monotherapy is perceived to have greater efficacy.

\section{BRAF inhibitor and MEK inhibitor combination therapy}

The most common cause of BRAF inhibitor monotherapy treatment failure is acquired resistance, with most patients experiencing disease progression with a median PFS of 6-8 months. Reactivation of the MAPK pathway is thought to be the main cause of acquired resistance, ${ }^{12,13}$ although MAPK-independent signalling through receptor tyrosine kinases has also been linked to the development of resistance. ${ }^{14,15}$

In preclinical studies, addition of a MEK inhibitor to a BRAF inhibitor decreased tumour growth, delayed the development of resistance and reduced the occurrence of skin lesions in metastatic melanoma models. ${ }^{16}$ As such, there has been considerable focus on combined therapy with dual BRAF and MEK inhibition as a means to improve outcomes compared with monotherapy (Table 1).

\section{Dabrafenib plus trametinib}

In a randomised open-label study of 162 patients with BRAFV600-mutant metastatic melanoma, median PFS in patients receiving dabrafenib plus trametinib in combination was 9.4 months compared with 5.8 months in patients treated with dabrafenib monotherapy (HR for progression or death, 0.39 ; $95 \% \mathrm{Cl}, 0.25-0.62 ; \mathrm{p}<0.001) .{ }^{17}$ Combination treatment was well tolerated with infrequent dose-limiting toxicities. In the subsequent phase III COMBI-d trial involving 423 previously untreated patients with stage IIIC-IV BRAF-mutant melanoma, median PFS was 9.3 months in patients randomised to dabrafenib plus trametinib versus 8.8 months 
in patients randomised to dabrafenib plus placebo (HR for progression or death $0.75 ; 95 \% \mathrm{Cl}, 0.57-0.99 ; \mathrm{p}=0.03){ }^{18}$ The overall response rate (ORR) was also significantly improved with combination treatment $(67 \%$ versus $51 \% ; p=0.002$ ). Adverse events were generally similar in the two groups, although the combined therapy group had a lower rate of cutaneous SCC (2\% versus $9 \%$ ) and a higher rate of pyrexia ( $51 \%$ versus $28 \%$ ) which was more often severe. In an update of these data, median PFS was 11.0 months (95\% $\mathrm{Cl} 8.0-13.9)$ in the dabrafenib and trametinib group and 8.8 months (5.9-9.3) in the dabrafenib group (HR $0.67,95 \% \mathrm{Cl} 0.53-0.84 ; \mathrm{p}=0.0004$ ). Median OS was also significantly longer with the combination; 25.1 months (95\% Cl 19.2-not reached) versus 18.7 months (15.2-23.7) with monotherapy (HR 0.71, 95\% Cl 0.55-0.92; $p=0.0107) .{ }^{19}$ One-, 2 - and 3-year Os rates were $74 \%, 51 \%$ and $44 \%$ in the combination group as compared with $68 \%, 42 \%$ and $32 \%$ in the dabrafenib group. The combination of dabrafenib and trametinib also provided significantly better health-related quality-of-life versus dabrafenib monotherapy..$^{20}$

The combination of dabrafenib plus trametinib was also assessed in the COMBI-v trial, in which it was compared with vemurafenib alone as firstline treatment in 704 BRAF-mutant patients with metastatic melanoma. ${ }^{21}$ ORR was significantly higher with dabrafenib plus trametinib compared to vemurafenib (67\% [95\% Cl: 62-72] versus 53\% [95\% Cl: 48-58]; $\mathrm{p}<0.001$ ) while median PFS was significantly longer (12.1 months versus 7.3 months; HR $0.61 ; 95 \% \mathrm{Cl}: 0.51-0.73, \mathrm{p}<0.001)$. HR for death in the dabrafenib plus trametinib group was 0.68 ( $95 \% \mathrm{Cl}, 0.56-0.83 ; \mathrm{p}=0.005$ ). Median OS was 26.1 months with combination therapy versus 17.8 months with vemurafenib alone. The survival benefit with dabrafenib plus trametinib was maintained at 2 and 3 years with, respectively, 53\% and $45 \%$ of patients receiving the combination treatment still alive compared with $39 \%$ and $31 \%$ of patients receiving vemurafenib alone. Significantly improved PFS was also maintained (12.6 months with dabrafenib plus trametinib versus 7.3 months with vemurafenib). Two- and 3-year PFS in the dabrafenib plus trametinib group was $30 \%$ and $24 \%$ compared with $16 \%$ and $10 \%$ in the vemurafenib group. ${ }^{22}$

\section{Vemurafenib and cobimetinib}

The combination of vemurafenib with cobimetinib, a potent, selective MEK1/2 inhibitor, has also been a focus of investigation. In the phase Ib BRIM-7 trial, 129 patients who had either recently progressed on vemurafenib or had never received a BRAF inhibitor were treated with combined vemurafenib and cobimetinib in a variety of dosing regimens (vemurafenib $720 \mathrm{mg}$ or $960 \mathrm{mg}$ twice a day continuously and cobimetinib $60 \mathrm{mg}$, $80 \mathrm{mg}$, or $100 \mathrm{mg}$ once a day for either 14 days on and 14 days off, 21 days on and 7 days off, or continuously). ${ }^{23}$ In BRAF inhibitor-naïve patients, the ORR was $87 \%$ and PFS was 13.7 months, although BRAF inhibitor pre-treated patients had a shorter PFS (2.8 months). Median PFS was 13.8 months ( $95 \% \mathrm{Cl} 10.1-20.6$ ) and median OS was 28.5 months (23.3-34.6) with a 2-year OS rate of $61 \%(95 \% \mathrm{Cl} 47.8-$ 74.4). ${ }^{24}$ The combination of vemurafenib and cobimetinib was generally well tolerated. The most frequent adverse events were similar to those with single agent monotherapy rash, (diarrhoea, photosensitivity and aspartate aminotransferase/alanine aminotransferase [ALT]) elevation, while there was a lower incidence of cutaneous SCC and KA.

Vemurafenib and cobimetinib combination therapy was further assessed in the CoBRIM trial, a multicentre, phase III study in which 495 patients with unresectable stage IIIC-IV BRAF-mutant melanoma were randomised to receive cobimetinib $60 \mathrm{mg}$ once daily with a 21 days on, 7 days off schedule plus vemurafenib 960 mg twice daily $(n=247)$ or vemurafenib plus placebo $(n=248) .{ }^{25}$ Treatment was continued until the occurrence of
Figure 1: Kaplan-Meier curve of overall survival in all patients

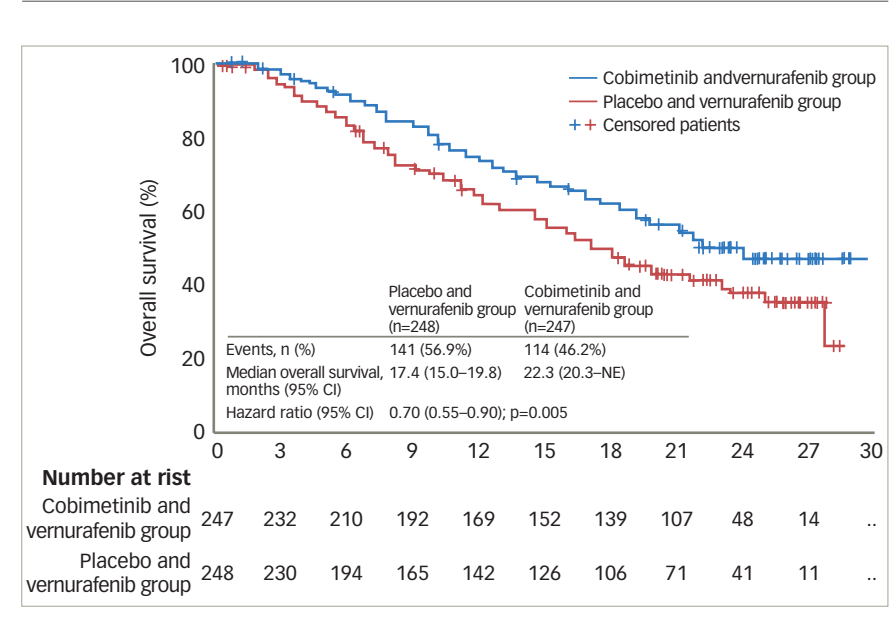

disease progression, unmanageable toxicity or withdrawal of consent. Investigator-assessed PFS was the study primary endpoint and OS, ORR and duration of response (DOR) were secondary end points.

After a median follow-up of 7.3 months, vemurafenib plus cobimetinib was associated with significantly longer median PFS compared with vemurafenib monotherapy ( 9.9 versus 6.2 months; HR for death or progression $0.51,95 \% \mathrm{Cl} 0.39-0.68 ; \mathrm{p}<0.001$ ). This benefit was observed in all pre-specified subgroups (disease stage, age, sex, geographic region, Eastern Cooperative Oncology Group [ECOG] performance status, lactate dehydrogenase [LDH] level, prior adjuvant therapy and BRAF mutation status). ORR was significantly improved with combination therapy compared with monotherapy ( $68 \%$ versus $45 \%$; $p<0.001$ ), with complete responses also more frequent (10\% versus $4 \%$ ). Median DOR was 7.3 months with vemurafenib but was not reached in the combination arm. Median OS was not reached at the time of the primary analysis, although analysis performed at the time of the final PFS analysis reported an OS rate of $81 \%$ for combination therapy compared to $73 \%$ with vemurafenib monotherapy (HR for death of $0.65,95 \% \mathrm{Cl} 0.42-1.00$; $p=0.046$; boundary $p<0.0000037$ ).

In an update to these data with median follow up of 14.2 months, the median PFS was 12.3 months $(95 \% \mathrm{Cl} 9.5-13.4)$ for vemurafenib plus cobimetinib versus 7.2 months (5.6-7.5) for vemurafenib alone (HR 0.58 [95\% Cl 0.46-0.72], p<0.0001). ${ }^{26}$ Median OS was 22.3 months $(95 \% \mathrm{Cl}$ 20.3-not reached) for vemurafenib and cobimetinib versus 17.4 months (95\% Cl 15.0-19.8) for placebo and vemurafenib (HR 0.70, 95\% Cl 0.55$0.90 ; \mathrm{p}=0.005$ ) (see Figure 1). One-year OS was $74.5 \%$ (95\% Cl 68.9-80.2) in the vemurafenib and cobimetinib group and $63.8 \%(57.6-70.0)$ in the vemurafenib group while 2-year OS was $48.3 \%$ (41.4-55.2) and $38.0 \%$ (31.3-44.7), respectively. ORR was similar to those at the primary analysis, although more patients achieved a complete response (16\% versus $10 \%$ in the vemurafenib and cobimetinib group and $11 \%$ versus $4 \%$ in the vemurafenib group), indicating that some patients had a better response when treatment was maintained.

Several tumour molecular markers were analysed at baseline to assess the possible effects of proliferative state (Ki67), MAPK activation (pERK) and pS6 (PI3K-MAPK downstream effector) on survival outcomes. ${ }^{26}$ High Ki67 expression was associated with a reduction in median OS in the vemurafenib group (HR 1.45,95\% Cl 0.94-2.25) but did not affect OS in the vemurafenib plus cobimetinib group. This might be a result of baseline Ki67 level being indicative of BRAF-MEK-ERK pathway activity and the increased effect of combined BRAF and MEK inhibition compared 
with BRAF inhibition alone. No associations were observed between clinical outcome and either PERK or pS6. This might be attributable to technical limitations in the immunohistochemistry analyses or possibly because other factors outweigh these signals. Although these findings require validation, they do suggest that survival of patients treated with combined BRAF and MEK inhibition might not be influenced by baseline tumour cell signalling status.

Tolerability was similar in the primary analysis and longer-term follow-up. Most of the side effects occurring with combination therapy were mild to moderate in severity (grade 1-2), although there was an increased incidence of grade $\geq 3$ treatment-related adverse events compared with vemurafenib monotherapy (60\% versus $52 \%$ ). ${ }^{26}$ The most frequent grade 3-4 adverse events that had a higher occurrence in the vemurafenib and cobimetinib group were increases in $\gamma$-glutamyl transferase, blood creatine phosphokinase (CPK) and ALT.

The frequency of cutaneous SCC, KA or Bowen's disease was reduced in the vemurafenib and cobimetinib group compared with the vemurafenib monotherapy group (6\% versus $20 \%$ of patients). Photosensitivity occurred more frequently in the vemurafenib and cobimetinib group, occurring in $34 \%$ of patients compared with $20 \%$ of patients in the vemurafenib group. However, most of these events were grade 1 and 2. Several MEK inhibitor-specific grade 2-3 events were observed, including serous retinopathy which was observed in $27 \%$ of patients in combination arm compared with $4 \%$ of patients with monotherapy. Again, most of these (90\%) were grade 1-2 and reversible without intervention. Patients in the vemurafenib and cobimetinib group also had similar quality of life compared with patients in the vemurafenib arm during the evaluable period (treatment cycles 1-8).

\section{Discussion}

The outcomes seen with combined vemurafenib and cobimetinib treatment in the COBRIM study are consistent with the results obtained with other studies that have assessed combined BRAF and MEK inhibition (i.e., trametinib and dabrafenib in the COMBI-d and COMBI-v studies). However, direct comparisons between trials are not feasible due to differences in patient characteristics and study designs e.g. the CoBRIM study did not allow crossover of patients from the vemurafenib group to the combination group with disease progression. In addition, PFS and ORR in the vemurafenib monotherapy arm were in line with results in previous randomised trials of vemurafenib monotherapy.,7

The CoBRIM trial enrolled a higher proportion of patients with elevated baseline LDH levels (around 50\%) compared with previous BRAF-MEK inhibitor studies. These patients have been shown to have a poorer prognosis in several trials., ${ }^{3,2}$ In the COMBI-v and COMBI-d trials, around $70 \%$ of patients had normal LDH levels, which might explain why patients receiving monotherapy in these studies had improved outcomes compared with previous studies (PFS of 7.6 months for vemurafenib and 8.8 months for dabrafenib compared with 6 months in initial phase II-III monotherapy trials). In the CoBRIM study, patients with raised LDH had shorter median survival than those with normal LDH levels in both treatment arms. These patients are often considered for treatment options other than BRAF or MEK-targeted therapies; however, they could benefit from long-term therapy with cobimetinib and vemurafenib.

In addition to targeted therapies, recent advances in the treatment and prognosis of advanced melanoma also include immune checkpoint inhibitors e.g. the cytotoxic T-lymphocyte-associated protein 4 (CTLA-4) inhibitor ipilimumab and the anti-PD-1/PD-L-1 antibodies nivolumab and pembrolizumab. Combined or sequential therapy involving targeted agents and immunotherapies is another area of considerable interest. However, a significant proportion of patients with disease progression on BRAF inhibitor monotherapy have rapidly progressive disease and a very poor prognosis. These patients typically do not survive long enough to complete ipilimumab treatment and so fail to receive the full benefit of immunotherapy. ${ }^{27,28}$ However, the use of combined BRAF/MEK inhibition in the CoBRIM trial seems be associated with a lower occurrence of rapidly-progressing disease. This may provide more opportunity to complete subsequent immunotherapy treatments.

A pooled analysis of 809 patients treated with dacarbazine, vemurafenib, or vemurafenib plus cobimetinib in the BRIM-2, BRIM-3, BRIM-7, and coBRIM studies was performed to identify patient subgroups with characteristics prognostic for post-progression OS (ppOS), defined as time from disease progression to death from any cause. A combination of $L D H$, disease stage at baseline, post-progression treatment, and ECOG performance status at progression of disease identified seven patient subgroups prognostic for ppOS. After adjusting for other covariates, postprogression treatment was associated with ppos, with similar results in the dacarbazine and vemurafenib cohorts. ${ }^{29}$

Vemurafenib plus cobimetinib was associated with an increased incidence of grade 3-4 toxicities compared with vemurafenib monotherapy, although discontinuation of therapy because of adverse events did not increase. MEK inhibitor-related adverse events, including diarrhoea, serous retinopathy, elevated CPK and increased liver enzyme levels, were frequent with the combination therapy, but resolved quickly and without treatment. Most toxicity was mild or moderate and occurred in the first 4 months of treatment. Ocular toxicity resolved in most patients without intervention and rarely resulted in cobimetinib treatment interruption or discontinuation. The dual inhibition of BRAF and MEK also reduced the incidence of cutaneous SCC/KA compared with vemurafenib alone (4\% versus $18 \%$ ).

On/off administration of cobimetinib when used in combination with vemurafenib may be of benefit in reducing toxicity. Ocular side effects were more manageable with an on/off schedule because in the seven 'off' days, patients generally recovered without treatment and could continue therapy without any dose reduction or interruption. The on/off blockade of BRAF signalling also appears to delay acquired resistance in preclinical studies. ${ }^{30}$

A study is now ongoing to evaluate the efficacy and safety of two different schedules of administration of vemurafenib in combination with cobimetinib (continuous and intermittent) in previously untreated patients with BRAFV600 mutation positive metastatic melanoma (NCT02583516).

So, considering the efficacy data about ORR and PFS emerging by the coBRIM trial, the safety profile and the possibility to complete a subsequent immunotherapy treatment, the combination of vemurafenib plus cobimetinib must be considered the standard of care for the first line treatment of patients with BRAF mutation positive metastatic melanoma.

\section{Conclusions}

The development of BRAF inhibitors represented a significant advance in the treatment of metastatic melanoma. However, recognition of the processes that underlie disease progression due to BRAF inhibitor resistance and the need to prolong the typical DOR has led to different combination approaches being assessed, including combined BRAF and MEK inhibition. In the COBRIM trial, vemurafenib and cobimetinib 
in combination resulted in improved in OS, PFS and ORR compared with vemurafenib monotherapy. This combination has recently been approved in Europe and the US for the treatment of advanced BRAFV600-mutant melanoma.

Dual inhibition of both MEK and BRAF appears to provide a more potent and durable anti-tumour effect than BRAF monotherapy, helping to prevent acquired resistance as well as decreasing toxicity secondary to BRAF inhibitor-induced MAPK-pathway activation. This may provide an increased possibility to complete subsequent sequential immunotherapy after disease progression. However, it should be noted that the majority of patients treated with the combination still experience disease progression. As such, further novel strategies involving immunotherapies and other targeted therapies are under investigation to further improve outcomes for patients with metastatic melanoma. Preliminary data from a phase $1 \mathrm{~b}$ study of the triple combination vemurafenib, cobimetinib and atezolizumab, a humanised engineered monoclonal antibody that targets PD-L-1, blocking interactions with PD-1 and B7.1, as treatment for untreated unresectable stage III/IV melanoma with V600E BRAF mutation were reported at the 2016 European Society of Medical
Oncology (ESMO) congress. Thirty patients were treated and the cohort that received vemurafenib plus cobimetinib run-in followed by the triple combination with atezolizumab, achieved a promising $83 \%$ ORR (10\% complete response and $72 \%$ partial response) with a good safety profile. ${ }^{31}$ The run-in phase with vemurafenib and cobimetinib in combination resulted very important due to the capability of the target therapy to recruit lymphocytes into the tumour, as demonstrated by the biopsies performed on tumour lesions before and after the treatment. These lymphocytes are then activated with the adding of atezolizumab to the treatment, improving the efficacy of the therapy. A phase III trial (TRILOGY -NCT02908672) has been designed to assess the efficacy of this triple combination as front-line treatment of patients with BRAF-mutated metastatic melanoma.

Considering the efficacy data and the safety profile, combined BRAF and MEK inhibition is the new standard of care in patients with advanced BRAF-mutant melanoma. This is not an arrival point, but represent the base to build new combinations with other anti-melanoma agents, as anti PD-1/PD-L-1 checkpoint inhibitors, to improve always more the prognosis of BRAF-mutated metastatic melanoma patients. $\square$
1. Hocker $\mathrm{T}, \mathrm{TsaO} \mathrm{H}$, Ultraviolet radiation and melanoma: a systematic review and analysis of reported sequence variants, Human Mutation, 2007;28:578-88.

2. Davies $\mathrm{H}, \mathrm{Bignell} \mathrm{GR}$, COX C, et al., Mutations of the BRAF gene in human cancer, Nature, 2002;417:949-54.

3. Chapman PB, Hauschild A, Robert C, et al., Improved survival with vemurafenib in melanoma with BRAF V600E mutation, N Engl J Med, 2011;364:2507-16.

4. McArthur GA, Chapman PB, Robert C, et al., Safety and efficacy of vemurafenib in BRAF(V600E) and BRAF(V600K) mutationpositive melanoma (BRIM-3): extended follow-up of a phase 3, randomized, open-label study, Lancet Oncol, 2014;15:323-32.

. Hauschild A, Grob JJ, Demidov LV, et al., Dabrafenib in BRAF-mutated metastatic melanoma: a multicentre, open-label, phase 3 randomized controlled trial, Lancet 2012;380:358-65.

6. Sosman JA, Kim KB, Schuchter L, et al., Survival in BRAF V600-mutant advanced melanoma treated with vemurafenib N Engl J Med, 2012;366:707-14.

7. Larkin J, Del Vecchio M, Ascierto PA, et al., Vemurafenib in patients with BRAF(V600) mutated metastatic melanoma: an open-label, multicentre, safety study, Lancet Oncol, 2014:15:436-44.

8. Oberholzer PA, Kee D, Dziunycz P, et al., RAS mutations are associated with the development of cutaneous squamous cell tumors in patients treated with RAF inhibitors, I Clin Oncol, 2012;30:316-21

9. Su F, Viros A, Milagre C, et al., RAS mutations in cutaneous squamous-cell carcinomas in patients treated with BRAF inhibitors, N Eng/ I Med, 2012;366:207-15.

10. Kim KB, Kefford R, Pavlick AC, et al., Phase II study of the MEK1/MEK2 inhibitor trametinib in patients with metastatic BRAF-mutant cutaneous melanoma previously treated with or without a BRAF inhibitor, I Clin Oncol, 2013;31:482-9.

11. Flaherty $\mathrm{KT}$, Robert $\mathrm{C}$, Hersey $\mathrm{P}$, et al., Improved survival with MEK inhibition in BRAF-mutated melanoma, N Engl I Med, 2012;367:107-14.

12. Wagle $\mathrm{N}$, Van Allen EM, Treacy DJ, et al., MAP kinase pathway alterations in BRAF-mutant melanoma patients with acquired resistance to combined RAF/MEK inhibition, Cancer Discov, 2014;4:61-8.

13. Shi H, Hugo W, Kong X, et al., Acquired resistance and clonal evolution in melanoma during BRAF inhibitor therapy, Cancer Discov, 2014;4:80-93

14. Rizos H, Menzies AM, Pupo GM, et al., BRAF inhibitor resistance mechanisms in metastatic melanoma: spectrum and clinical impact Clin Cancer Res, 2014:20:1965-77.

15. Van Allen EM, Wagle N, Sucker A, et al., The genetic landscape of clinical resistance to RAF inhibition in metastatic melanoma Cancer Discov, 2014;4:94-109

16. King AJ, Arnone MR, Bleam MR, et al., Dabrafenib; preclinical characterization, increased efficacy when combined with trametinib, while BRAF/MEK tool combination reduced skin lesions, PLoS One, 2013;8:e67583.

17. Flaherty KT, Infante JR, Daud A, et al., Combined BRAF and MEK inhibition in melanoma with BRAF V600 mutations, N Eng/ Med, 2012;367:1694-703.

18. Long GV, Stroyakoversuskiy D, Gogas $\mathrm{H}$, et al., Combined BRAF and MEK inhibition versus BRAF inhibition alone in melanoma, N Engl J Med, 2014;371:1877-88.

19. Long GV, Stroyakoversuskiy D, Gogas $\mathrm{H}$, et al., Dabrafenib and trametinib versus dabrafenib and placebo for Val 600 BRAF-mutant melanoma: a multicentre, double-blind, phase 3 BRAF-mutant melanoma: a multicentre, double-blind,

20. Flaherty K, Davies MA, Grob JJ, et al., Genomic analysis and 3-y efficacy and safety update of COMBI-d: A phase 3 study of dabrafenib (D) + trametinib (T) vs D monotherapy in patients (pts) with unresectable or metastatic BRAF V600E/K-mutant cutaneous melanoma, J Clin Oncol, 2016; 34(Suppl):abstr 9502

21. Robert C, Karaszewska B, Schachter J, et al., Improved overall survival in melanoma with combined dabrafenib and trametinib, N Eng/ J Med, 2015;372:30-9.

22. Robert C, Karaszewska B, Schachter J, et al., Three-year estimate of overall survival in COMBI-V, a randomized phase 3 study evaluating first-line dabrafenib (D) + trametinib (T) in patients (pts) with unresectable or metastatic BRAF V600E/Kmutant cutaneous melanoma, Ann Oncol, 2016;27(Suppl 6): LBA40.
23. Ribas A, Gonzalez R, Pavlick A, et al., Combination of vemurafenib and cobimetinib in patients with advanced BRAF(V600)-mutated melanoma: a phase 1b study, Lancet Oncol, 2014;15:954-65.

24. Pavlick AC, Ribas A, Gonzalez R, Hamid O, Extended follow-up results of phase Ib study (BRIM7) of vemurafenib (VEM) with cobimetinib (COBI) in BRAF-mutant melanoma, J Clin Oncol, cobimetinib (COBI) in BRAF-m

25. Larkin J, Ascierto PA, Dreno B, et al., Combined vemurafenib and cobimetinib in BRAF-mutated melanoma, N Eng/ I Med, 2014;371:1867-76.

26. Ascierto PA, MCArthur GA, Dréno B, et al., Cobimetinib combined with vemurafenib in advanced BRAF(V600)mutant melanoma (COBRIM): updated efficacy results from a randomized, double-blind, phase 3 trial, Lancet Oncol, 2016;17:1248-60

27. Ascierto PA, Simeone E, Giannarelli D, et al., Sequencing of BRAF inhibitors and ipilimumab in patients with metastatic melanoma: a possible algorithm for clinical use, I Trans/ Med 2012;10:107.

28. Ascierto PA, Simeone E, Sileni VC, et al., Sequentia treatment with ipilimumab and BRAF inhibitors in patients with metastatic melanoma: data from the Italian cohort of the ipilimumab expanded access program, Cancer Invest, 2014:32:144-9.

29. Ascierto P, Ribas T, Larkin J, et al., Prognostic subgroups and impact of treatment for post-progression overall survival (ppOS) in patients (pts) with BRAFV600-mutated metastatic melanoma treated with dacarbazine (DTIC) or vemurafenib (VEM) \pm cobimetinib (COBI): A pooled analysis, Ann Oncol, 2016;27(Suppl 6):vi394

30. Das Thakur M, Salangsang F, Landman AS, et al., Modelling vemurafenib resistance in melanoma reveals a strategy to forestall drug resistance, Nature, 2013;494:251-5.

31. Hwu P, Hamid o, Gonzalez R, et al., Preliminary safety and clinical activity of atezolizumab combined with cobimetinib and vemurafenib in BRAF V600-mutant metastatic melanoma Ann Oncol, 2016;27(Suppl 6):vi381. 